\section{Understanding racial-ethnic and societal differentials in STI}

\section{SO Aral}

\section{Do we need to move beyond behavioural epidemiology?}

$\mathrm{P}$ revalence and incidence of sexually transmitted infections (STIs) vary across societies ${ }^{1}$ and across subpopulations defined by age, raceethnicity, and socioeconomic status. ${ }^{23}$ The efforts to account for such variation and explain it, that can be found in the STD literature, have in general not differentiated between individual and population level health, or between population and individual level determinants of individual STD outcomes. ${ }^{4}$ Perhaps this pattern reflects the predominant paradigm in modern epidemiology which has been termed the "risk factor" paradigm and has been linked to "biomedical individualism" as its underlying theoretical foundation. ${ }^{56}$ This theoretical approach views populations simply as reflective of individual cases while considering social determinants of disease to be at best secondary, if not irrelevant. ${ }^{\text {? }}$ In the past several years, the risk factor paradigm in epidemiology has been seriously challenged by leading epidemiologists ${ }^{89}$ and a new paradigm that would emphasise the broader context of individual risk factors has been called for. It has been suggested that whereas traditional epidemiologists ask the question "Why are some individuals healthy and others not?" the social epidemiologist is concerned with the question "Why are some societies healthy while others are not?"10 Social epidemiology has focused on features of the economy, culture, politics, and the law. Examples of societal characteristics that have received attention include macroeconomic factors such as poverty, unemployment, and income distribution; and features of social relationships such as social cohesion, social exclusion, and sex and race relationships. ${ }^{11}$ Also, a renewed interest in effects of neighbourhood environments on morbidity and mortality has emerged. ${ }^{12-14}$

Work in social epidemiology has emphasised neighbourhoods and the community; and considerations of social capital and collective efficacy have usually been applied to chronic diseases, mortality, violence, and mental health as health outcomes. Infectious diseases and, particularly, their modes of transmission are often ignored in this litera- ture. Moreover, the social epidemiological approach is often successful in the description of social correlates of morbidity and mortality; identification of mechanisms of action through which social determinants influence levels and distribution of morbidity in populations tends to be more difficult.

Social epidemiological approaches may have a lot to offer to the explanation of STD differentials within and across societies. Conversely, STD epidemiology, with its distinct transmission dynamics may provide detailed examples of mechanisms of action through which social determinants operate. What follows is a description of one possible way in which relations among social determinants, their mechanisms of action, and their impact on STD morbidity may be conceptualised.

\section{THE SOCIAL NETWORKS APPROACH IN STD EPIDEMIOLOGY}

During the past decade an important trend in STD epidemiology research has focused on the role of sexual networks in the spread of STIs in populations. Findings demonstrated that in the United States, the higher rates of sexual contact between the "core" group and the "periphery" among African Americans facilitate the spread of infection overflow into the African American general population; whereas the "sexual segregation" of African Americans from other racialethnic groups results in STIs remaining inside this population. ${ }^{15}{ }^{16}$ Other studies have shown that linkages between sexual networks are necessary for the spread of STIs across sexual networks ${ }^{17}$; that so called "core groups" appear to be important in the spread of STIs and in their prevention ${ }^{18-20}$; and that the sexual transmission of sexually transmitted diseases (STDs) and HIV beyond core groups may depend on people who have sexual intercourse with members of core groups and with members of the general population-so called "bridge populations." ${ }^{17}$ Studies in Thailand and other populations revealed that large proportions of men in certain occupations, such as truck drivers, the police, and the military tend to function as "bridges" between female sex workers and their wives or girlfriends. ${ }^{21}{ }^{22}$ However, one study conducted in Seattle found the proportion of infection attributable to bridge populations to be remarkably small; with most of the disease burden for gonococcal and chlamydial infections in both high prevalence and low prevalence subpopulations being attributable to mixing within the subpopulation or to direct mixing with members of high prevalence subpopulations..$^{23}$ It appears that bridge populations play a very important part in the introduction of infection into subpopulations, once the infection is introduced, most of the disease burden is attributable to mixing within the subpopulation.

Other studies conducted in Canada reveal that sexual network patterns involved in STD epidemics vary across phases of epidemics ${ }^{24}$ and that during later phases of STD epidemics, the majority of sexual networks involved in the epidemic are not restricted to one geographic area ${ }^{25}$; frequent contact between network members from a small group of northern reserves and individuals in the major southern population centre of Winnipeg have formed bridges of transmission between these communities. Similarly, a study of elimination and reintroduction of primary and secondary syphilis in Seattle showed that characteristics of persons with primary and secondary syphilis varied across epidemic spread, elimination, and reintroduction periods. ${ }^{26}$ There were significant differences between cases during the various epidemic phases with respect to age, sex, ethnicity, drug use, and involvement with commercial and anonymous sex. Moreover, during all phases, imported cases differed from locally acquired cases with respect to age, sex, ethnicity, and drug use behaviours.

One network pattern that has received increasing research attention in recent years is concurrent partnerships, or sexual partnerships that overlap over time. ${ }^{27}$ Concurrent partnerships accelerate the spread of an STI through a population by removing the protective factors of time and sequence inherent in serial monogamy.

Sexual networks and patterns of sexual partnership formation and dissolution constitute a major mechanism of action through which the political economy and the sociolegal system influence the rate of spread of STI in a population; availability, accessibility, and utilisation of appropriate health care, and availability and utilisation of condoms being others.

\section{CREATION, MAINTENANCE, AND} EVOLUTION OF CORE GROUPS

Sexual networks that are highly critical to the rate of spread of STI include sex 
work; exchange of sex for gifts, material needs or drugs; and anonymous sex. The creation, maintenance, and expansion of sex in exchange for money or other goods appears to be highly sensitive to changes in political economy and the sociolegal system. Internal conflicts, war, economic crises, and social collapse are accompanied by the establishment of major sex markets or the expansion of existing ones. For example, in Moscow, Russia, before the August 1998 economic crisis the number of female sex workers was estimated at 15 000-30 000; following the crisis this number increased to $30000-90000 .^{28}$ Similarly, in Jakarta and Surabaya, Indonesia, between 1997 and 1998-during which time the monetary crisis occurred-the percentage of female sex workers who were less than 20 years of age increased by $38 \%$ and $125 \%$ respectively; the percentage of female sex workers with less than 12 months' experience increased by $28 \%$ and $130 \%$ during the same period in these two cities. ${ }^{29} \mathrm{~A}$ decade of conflict in the Balkan region and the poverty of post communist eastern Europe have created a major network of trafficking in women which reaches across eastern Europe, Balkans, the Middle East, and Western Europe. ${ }^{30}$ Globalisation, characterising many social, economic, and behavioural patterns, includes sex work and expands the volume of sex workers. $^{31} 32$

The political economy and the sociolegal system and any changes in them systematically impact lower socioeconomic status, minority groups to a greater extent than others. Sociolegal systems tend to reinforce existing hierarchies and protect the privileged. Thus, social parameters often create the context which facilitates the creation, maintenance, and expansion of sexual networks that cause rapid spread of STD among minority racial, ethnic, and socioeconomic groups and in less developed societies. The same context associates longer duration of infection with the less privileged groups and less privileged societies through differential access to, and differential utilisation of, good quality STD care. A detailed description of the sociopolitical context in which African Americans live in the rural southeastern United States powerfully elucidates how contextual features including racism, discrimination, limited employment opportunity, and resultant social and economic inequity may promote sexual network patterns that transmit STIs. ${ }^{33}$

Despite the powerful effects of social context and social determinants embedded in political economy and sociolegal systems on the level and distribution of STDs, most of our research and surveillance attention focuses on the STDs themselves and to a limited extent on the individual behaviours associated with STD acquisition. It is important to develop standard techniques for summarising the extent and nature of sex work within populations and for determining the size and nature of the interactions among core groups, bridge populations, and the general population. Moreover, most research and surveillance activity tends to be conducted within local boundaries, without much attention to sexual links that relate many local sexual networks to each other. Given that prevalence of sex work seems dependent on global forces, and that sex workers seem to be highly mobile across geographic areas, it may be important to consider the establishment of surveillance systems that include monitoring of contextual parameters and social determinants at regional and global levels. ${ }^{34}$ STD prevention programmes may be more effective if informed about the local, regional, and global context in which STD rates rise and fall; and which create the inequalities in STD incidence across societies and across groups defined by race-ethnicity and socioeconomic status.

\section{ACKNOWLEDGEMENTS}

The author acknowledges Ms Patricia Jackson for her outstanding support in the preparation of this manuscript.

Sex Transm Inf 2002;78:2-4

\section{Author's affiliations}

S O Aral, Centers for Disease Control and Prevention, National Center for HIV, STD and TB Prevention, Division of STD Prevention, Atlanta, GA, USA

Correspondence to: Division of STD Prevention, CDC, 1600 Clifton Road, NE, M/S-E02, Atlanta, GA 30333, USA; pbj9@cdc.gov

\section{REFERENCES}

1 Aral SO, Holmes KK. Epidemiology of sexual behavior and sexually transmitted diseases. In: Holmes KK, Mardh P-A, Sparling PF, Wiesner PJ, Cates Jr W, Lemon SM, Stamm WE, eds. Sexually transmitted diseases. 2nd ed. New York: McGraw Hill, 1990:19-36.

2 Fenton K, Johnson AM, Nicoll A. Race, ethnicity and sexual health. BM 1997;314:1703-4

3 Evans BA, Kell PD, Bond RA, et al. Racial origin, sexual lifestyle, and genital infection in London (1992). Sex Transm Inf 1998;74:45-9.

4 Aral SO, Holmes KK, NS Padian, et al. Overview: individual and population approaches to the epidemiology and prevention of sexually transmitted diseases and human immunodeficiency virus infection. $J$ Infect Dis 1996;174(Suppl 2): 127-33.

5 Schwartz S, Susser E, Susser M. A future for epidemiology? Annu Rev Public Health 1999:20:15-33.

6 Diez-Roux, Ana V. Bringing context back into epidemiology: variables and fallacies in multilevel analysis. Am J Public Health 1998;88:216-22.

7 Krieger N. Epidemiology and the web of causation: has anyone seen the spider? Soc Sci Med 1994;39:887-903.
8 Shy CM. The failure of academic epidemiology: witness for the prosecution. Am J Epidemiol 1997; 145:479-87.

9 Schwartz S. The fallacy of the ecological fallacy: the potential misuse of a concept and the consequences. Am J Public Health 1994;84:819-24.

10 Kawachi I, Burris S, Sarat A, eds. The epidemiology of law. In: Health, law and human rights: exploring the connection. (In press)

11 Kawachi I, Berkman LF. Social cohesion, social capital and health. In: Berkman LF, Kawachi I, eds. Social epidemiology. New York: Oxford University Press, 2000.

12 Stephanie R. Socioeconomic position and health: the independent contribution of community socioeconomic context. Annu Rev Sociol 1999;25:489-516.

13 Yen IH, Syme SL. The social environment and health: a discussion of the epidemiologic literature. Annu Rev Public Health 1999;20:287-308

14 Kawachi I, Kennedy BP, Wilkinson RG. The society and population health reader: income inequality and health. New York: The New York Press, 2000

15 Laumann EO, Youm Y. Racial/ethnic group differences in the prevalence of sexually transmitted diseases in the United States: a network explanation. Sex Transm Dis 1999;26:250-61.

16 Aral SO. Sexual network patterns as determinants of STD rates: paradigm shift in the behavioral epidemiology of STDs made visible (editorial). Sex Transm Dis 1999;26:262-4.

17 Anderson R, May R, Boily N, et al. The spread of HIV-I in Africa: sexual contact patterns and the predicted demographic impact of AIDS. Nature 1991;352:581-9.

18 Thomas JC, Tucker M. The development and use of the concept of a sexually transmitted disease core. J Infect Dis 1996;174(Suppl 2): 134-43.

19 Steen R, Vuysteke B, DeCoito T, et al. Evidence of declining STD prevalence in a South African mining community following core group intervention. Sex Transm Dis 2000; 27: 1-8.

20 VanDam CJ, Holmes KK. STD prevention: effectively reaching the core and a bridge population with a four-component intervention. Sex Transm Dis 2000;27:9-11.

21 Morris M, Podhisita C, Waver M, et al. Bridge populations in the spread of HIV/AIDS in Thailand. AIDS 1996;10:1265-71.

22 Gorbach PM, Sopheab H, Phalla T, et al. Sexual bridging by Cambodian men: potential importance for general population spread of STD/HIV epidemics. Sex Transm Dis 2000;27:320-6.

23 Aral SO, Hughes JP, Stoner B, et al. Sexual mixing patterns in the spread of gonococcal and chlamydial infections. Am J Public Health 1999:89:825-33.

24 Wasserheit JN, Aral SO. The dynamic topology of sexually transmitted disease epidemics: implications for prevention strategies. J Infect Dis 1996;174(Suppl 2):S201-13.

25 Jolly AM, Matusho P, Wylie J. Sexual contact networks and chlamydia transmission in Manitoba, Canada. Paper presented at Sunbelt XIX. International Sunbelt Social Network Conference; 18-21 February 1999. Charleston, SC

26 Williams LA, Klausner JD, Whittington WLH, Handsfield HH, Celum C, Holmes KK. Elimination and reintroduction of primary and secondary syphilis. Am J Public Health 1999;89:1093-7.

27 Morris M, Kretzchmar M. Concurrent partnerships and transmission dynamics in networks. Social Networks 1995; 17:299-318.

28 R Montgomery. Presented at the XIII International AIDS Conference, Durban, South Africa, July 2000

29 Moran J. Indonesian monetary crisis and the commercial sex industry; effects on volume, supply and demand. Unpublished report. 
Division of STD Prevention, CDC, Atlanta, Georgia. May 2000

30 Gall C. Macedonia village is center of Europe web in sex trade. New York Times 2001 July 28

31 Koenig ER. International prostitutes and transmission of HIV. Lancet 1989;1:782-3.
32 Report on International workshop on trafficking in women to and from Central and Easter Europe. Budapest, Hungary, 4-5 October 1996.

33 Adimora AA, Schoenbach VJ, Martinson FEA, et al. Social context of sexual relationships among rural African Americans. Sex Transm Dis 2001;28:69-76.

34 Aral SK, Berman SM, Aral SO. Anticipating outbreaks: a prevention role for integrated information systems. Sex Transm Dis 2002;29:6-12.
Vaccination

\section{Preventive human papillomavirus vaccination}

\section{Lehtinen, J Dillner}

\section{Considerable gains at the individual and societal level would} be obtained if cervical cancer could be prevented

$\mathrm{T}$ he cancer burden causally associated with human papillomavirus (HPV)

infections is high. Cervical cancer is the second most common cancer among females in the world, with 500000 new cases and 300000 premature deaths a year. ${ }^{1}$ Because of the long preclinical period cervical cancer can be prevented by screening, diagnosis, and treatment of premalignant cervical lesions, but for developing countries preventive vaccination may be the only possibility to significantly reduce cervical cancer incidence. Also in the developed countries considerable gains at the individual and societal level would be obtained, if a significant proportion of cervical cancer and its precursor lesions could be prevented by HPV vaccination (for a systematic review see Lehtinen et $a l^{2}$ ). In addition, other anogenital cancers, oropharyngeal and base of tongue cancers, and probably a small proportion of oesophageal cancers are all strongly associated with past HPV infection. ${ }^{3-5}$ For these and other possible HPV associated cancers, vaccination may be the only possibility for prevention. Overall prevention of HPV infections may result in a $5-10 \%$ reduction of cancer mortality worldwide. This editorial seeks to answer the following two questions: what kind of vaccines will be tested and how should their efficacy be defined?

Preventive HPV vaccines entering clinical efficacy (phase III) trials are plain virus-like particles (VLPs), DNA free capsids comprising the major viral capsid (Ll) protein (manufactured by Merck, GlaxoSmithKline, and by NIH), or chimeric VLPs (CVLP), containing various combinations of early viral proteins attached in different ways to the major Ll or the minor (L2) capsid proteins of the virus.
In phase I and II trials HPV VLPs have proved to be safe and highly immunogenic. ${ }^{6}$ HPV VLP immunisation induces approximately 100-fold higher neutralising antibody titres than natural infection. The level of mucosal immunoglobulin $\mathrm{G}(\operatorname{Ig} G)$ is $10 \%$ but it varies following the menstrual cycle and is lowest at the time of ovulation. The prevailing theory of the mode of action of the vaccine, however, suggests that this variation may not be a major problem. In natural infection the entry of HPV into the basal cells of the epithelium, which support the initial stages of viral replication, is facilitated by a microscopic trauma resulting from, for example, sexual intercourse. Following this micro trauma, circulating antibodies leak to the epithelial surface and neutralise the virus.

The $\mathrm{Ll}$ antibodies recognise a conformational, type specific epitope, and have shown close to a $100 \%$ protection in animal studies against homologous challenges with both HPV and animal papillomaviruses. ${ }^{7}$ While the increasingly large number of oncogenic HPV types $(16,18,31,33,35,45,51,52,58,59)$ that associate with cervical cancer may make it impossible to achieve $100 \%$ protection against cervical cancer, it is relatively easy to include the most prevalent oncogenic HPVs (HPVl6 and HPV18) into a multivalent VLP vaccine, and even tailor the vaccine composition by the HPV types most prevalent in different geographic areas should this prove necessary.

Analogously to hepatitis B virus (HBV) vaccine HPV VLPs also induce cytotoxic $\mathrm{T}$ cell (CTL) responses by entering the MHC class I pathway. ${ }^{7}$ If the antibodies fail to neutralise all HPV virions, CTLs recognising viral capsids bound for as long as 10-12 hours to more or less specific cellular receptors (integrin and/or heparan sulphate proteoglycans ${ }^{89}$ ) might block spread of the virus at its most primordial state in the initially infected cells. Production of new virions takes place in the upper layers of the epithelium, and CTLs targeting these cells might effectively reduce spread of the virus. Indication of this has, however, been shown only for the non-oncogenic HPV VLPs, and it is not clear whether such a response is able to eliminate oncogenic HPVs from the basal cells. ${ }^{10}$

CVLPs may offer a significant advantage in this regard. The expression of various early HPV proteins responsible for viral replication (El), transcription (E2), and oncogenesis (E6, E7) is abundant both in the basal and the differentiating epithelial cells providing good targets for the CTLs. Two vaccines based on different gene constructs-HPV16 Ll, L2 truncated E2-E7 CVLP (by an NIH group) and HPVl6 L1-E7 CVLP (by Medigene)-have passed or are passing safety and immunogenicity tests. In addition to the induction of high titres of neutralising antibodies, some of which (anti-L2 antibodies) may be cross protective against several HPV types, the CVLP vaccines induce CTL responses against the early proteins in humans. ${ }^{11}$ However, for CVLPs data on humans are scarce and need to be expanded. CTL responses against the early HPV proteins are important not only in order to provide theoretically improved protection and possible therapeutic effect, but because they may also offer cross protection against several HPV types. The E1 and E2 proteins are particularly well conserved among the HPVs.

The analogy between the different HPV VLP vaccines and the first human cancer vaccine, HBV vaccine, is very encouraging. The HBV vaccine has an overall efficacy of $95 \%,{ }^{12}$ and even when given to infants born to mothers with active hepatitis (HBV-e antigen positive women, the offspring of whom are prone to become chronic HBV carriers) its efficacy exceeds $75 \%$. These figures also fit the first available data on long term effects of universal $\mathrm{HBV}$ vaccination. The incidence of liver cancer has reduced by $75 \%$ among 12-14 year old Taiwanese children 15 years after implementation of the nationwide HBV vaccination programme. ${ }^{13}$ This was to be expected on the basis of seroepidemiological data showing that HBs antibody positive 
individuals have a reduced risk of liver cancer, whereas HBs antigen positive individuals have an increased risk. This was the first randomised trial proving the efficacy of $\mathrm{HBV}$ vaccination against both acute hepatitis $\mathrm{B}$ and becoming a chronic HBV carrier. But, however encouraging, analogies should be considered with caution.

While it appears that most of the women treated for the HPV induced premalignant lesions by, for example, laser or loop excision can tackle the residual low amount of virus and eventually clear the infection, recurrences do occur with varying incubation times and for reasons that are not totally understood. ${ }^{14}$ Restricting the viral load plays a part, and new strategies for cervical cancer control are also based on identification of women with moderate to high levels but not low levels of oncogenic HPV DNA (for a systematic review see Cuzick et $\left.a l^{15}\right)$. The role of natural infection or vaccine induced VLP antibodies in restricting mucosal HPV infection may, however, be qualitatively different from the central role of circulating HBV antibodies in preventing systemic hepatitis B infection. While HBV antibody positive individuals have a reduced risk of liver cancer, the HPV16 VLP antibody positive individuals remain at an increased risk of developing cervical cancer and other HPVI6 associated cancers 10-20 years after infection..$^{2-5}$ There are no good data to suggest that the antibodies would do any harm-for example, by inducing latency, but we simply do not know to what extent the paradigm on prevention of liver cancer by preventing acute HBV infection and HBV carrier status can be applied in the HPV infection-cervical neoplasia context.

The main effector function of VLP vaccination is neutralising antibodies ${ }^{7}$ but these may never be able to induce totally sterilising immunity and the concept of significantly reducing the viral load becomes an issue. ${ }^{16}$ While the minimum HPV viral load for development of cervical and other cancers is not known, it is highly likely that HPV vaccine induced neutralising antibodies and/or CTLs will prevent or significantly restrict and aid in clearing of the primary HPV infection, and reduce transmission of the infection to others. Randomised clinical trials will eventually define efficacy of the different vaccines against persistent HPV infection and other surrogate end points, such as cervical intraepithelial neoplasia grade II/III. A proof of the principle that HPV vaccines can prevent these necessary steps in cervical carcinogenesis might be considered sufficient to demonstrate efficacy and to compare different vaccines, and limited licensures will be considered probably sooner rather than later.

There are, however, several possible pitfalls that could prevent effective vac- cines from actually achieving their expected health benefits. For example, if vaccination failure is preferentially associated with determinants of progression or if vaccination induces changes in the population biology of the different HPV types. To find out these pieces of information one has to organise a long term follow up of the initial randomised trials. Countries with stable and vaccination prone populations, population based health registers, standardised public health care, and organised mass screening for cervical cancer have the appropriate infrastructure and setting for direct extension of the clinical trials to the invasive cervical cancer (ICC) and cervical intraepithelial neoplasia grade III (CINIII) end points based on registry follow up. ${ }^{17}$ Population based randomisation and informed consent based linkages of the different study and health registers from the very beginning to death are most important especially to avoid different selection biases, performance bias resulting from "contamination" of the population after licensure of the vaccines, ${ }^{18}$ and loss to follow up bias. The ultimate proof will be that immunisation with HPV vaccines significantly reduces the incidence of (and mortality from) cervical cancer and its immediate precursors compared to unvaccinated population based referents. ${ }^{17}$ If it turns out that the plain VLP vaccines fail to do it, while the chimeric VLP vaccines are successful we can infer that simple reduction of the HPV load at the port of viral entry is not enough, and that the second barrier of cell mediated immunity against the early viral proteins is also needed. At the moment, however, there is no indication of this and both alternatives should be pursued.

For all end points it would be optimal to target large numbers of young boys and girls who are about to start their sexual activity, since they will have the highest event rates of both HPV infections and associated cancers. Targeting both sexes may, however, not be necessary at this stage since the assumed vaccine efficacy against HPV infection $(90 \%)$ is high enough to bring the beneficial long term effect to the females. ${ }^{19}$ With an assumed attack rate of $0.65 \%$ and $60 \%$ vaccine efficacy against CINIII+ICC, enrolment of altogether 15000 such vaccinees and referents for 15-20 years of registry based follow up would give $80 \%$ statistical power to judge whether a vaccine which covers two thirds of the oncogenic HPV types protects against CINIII+ICC. Comparison of different HPV vaccines and gradual implementation of the adolescent vaccination into the general vaccination programme using the same setting would bring in considerable synergy and needs to be considered seriously. Last but not least, possible ethical prob-

\section{Key messages}

- DNA free virus-like particle vaccines containing either structural (L1) HPV proteins or both structural and early viral proteins have passed/are passing safety/immunogenicity trials.

- Phase III efficacy trials with the different VLP vaccines using intermediate end points such as protection against HPV positive squamous intraepithelial lesions will start soon.

- Long term follow up of the phase III trials would be important to obtain proof for the vaccine efficacy against cervical cancer.

- Phase III and the long term follow up trials may help future public health policy decision making.

lems associated with ending or discontinuing the early end point clinical trials would be largely solved by the possibility of referring the vaccinees to an organised mass screening.

Pieces of the preventive HPV vaccination puzzle are on the table. If the scientific community, together with the public health authorities and vaccine manufacturers, manages to solve the puzzle the expected health benefits are immense.

\section{ACKNOWLEDGEMENTS}

The authors wish to thank Drs Maurice Hilleman and Eero Pukkala for stimulating discussions.

Sex Transm Inf 2002;78:4-6

\section{REFERENCES}

1 Parkin DM, Pisani P, Ferlay J. Estimates of the worldwide incidence of 25 major cancers in 1990. Int J Cancer 1999;80:827-41.

2 Lehtinen M, Luukkaala T, Dillner J, et al. Human papillomavirus type 16 infection, subsequent risk for cervical neoplasia and associated population attributable fraction. A systematic review. J Clin Virol 2001;22:113-22.

3 Dillner J, Hakulinen T, Knekt $P$, et al. Prospective seroepidemiological evidence that human papillomavirus type 16 infection is a risk factor for oesophageal squamous cell carcinoma. BM 1995;311:1346.

4 Bjorge T, Dillner J, Anttila T, et al. Prospective seroepidemiological study of the role of human papillomavirus in non-cervical anogenital cancers. BM 1997;315:646-9 5 Mork J, Lie AK, Glattre E, et al. Human papillomavirus infection as a risk factor for squamous-cell carcinoma of the head and neck. N Engl J Med 2001;344:1125-31.

6 Harro CD, Susana Pang Y-Y, Roden RBS, et al. Safety and immunogenicity trial in adult volunteers of a human papillomavirus $16 \mathrm{~L} 1$ virus-like particle vaccine. I Natl Cancer Inst 2001;93:284-92.

7 Schiller J, Hildesheim A. Developing HPV virus-like-particle vaccines to prevent cervical cancer: a progress report. J Clin Virol 2000;19:67-74

8 Evander M, Frazer IH, Payne E, et al. Identification of the alpha (6) integrin as a candidate receptor for papillomaviruses. J Virol 1997;71:2449-56.

9 Giroglou T, Sapp M, Lane C, et al. Immunological analyses of human 
papillomavirus capsids. Vaccine 2001;19:1783-93.

10 Lehtinen M, Paavonen J. Efficacy of preventive HPV vaccination. Int J STD AIDS 2001;12:771-6.

11 Kaufmann AM, Nieland J, Schinz M, et al. HPV 16 L1E7 chimeric virus-like particles induce specific HLA-restricted T cells in humans after in vitro vaccination. Int J Cancer 2001:92:285-93.

12 Hilleman MR. Overview of the pathogenesis, prophylaxis and therapeusis of viral hepatitis $B$, with focus on reduction to practical applications. Vaccine 2001;19:1837-48.

13 Huang KY, Lin S-R. Nationwide vaccination: a success story in Taiwan. Vaccine 2000; 18:535-8

14 Elfgren K, Bistoletti P, Dillner L, et al Conization for cervical intraepithelial neoplasia is followed by disappearance of human papillomavirus deoxyribonucleic acid and a decline in serum and cervical mucus antibodies against human papillomavirus antigens Am J Obstet Gynecol 1996;174:937-42.

15 Cuzick J, Sasieni P, Davies P, et al. A systematic review of the role of human papillomavirus testing within a cervical screening programme. Health Technol Assess 1999;3:1-196.

16 Zinkernagel RM, LaMarre A, Ciurea A, et al. Neutralizing antiviral antibody responses. $A d v$ Immunol 2001;79: 1-53.

17 Lehtinen $M$, Kibur $M$, Luostarinen $T$, et al. Prospects for phase III-IV HPV vaccination trials in the Nordic countries and in Estonia. J Clin Virol 2000;19:113-22.
18 Juni $\mathbf{P}$, Altman D, Egger M. Assessing quality of controlled clinical trials. BM $2001 ; 323: 442-6$

19 Garnett GP, Waddell H. Public health paradoxes and the epidemiological impact of an HPV vaccine. J Clin Virol 2000;19:101-12.

\section{Authors' affiliations}

M Lehtinen, J Dillner, National Public Health Institute, Helsinki, Finland, and Department of Medical Microbiology, University of Lund,

Malmö, Sweden

Correspondence to: Dr M Lehtinen, Department of Infectious Disease Epidemiology,

Mannerheimintie 166, Fl 00300 Helsinki,

Finland; Ilmale@uta.fi

Editorial

\section{The year ahead}

\section{Mohsen Shahmanesh}

W have introduced some new sections with the aim of adding value to the journal. Update has replaced the Recent Publications section and hopes to bring expert critical summary of topic based important recent publications-pelvic inflammatory disease on this occasion. An impending tropical disease section, edited by David Lewis, will provide state of the art summaries of diagnosis and management of these conditions, embracing also issues faced in resource poor settings.

Later this year we will begin our interactive CME section, based on "grey cases." Sarah Edwards will be heading this section helped by Richard Lau. We are negotiating with the Royal College of Physicians to gain CPD recognition. Our expanded editorial board have all promised to provide us with either an Update or a review article, and we are waiting for these to roll in.

\section{IMPACT FACTOR}

Finally to the issue of impact factor, with which our funding authorities appear so infatuated. After disappearing into the ether as a result of our name change we have re-emerged with an unprecedented factor of 2.1 (fig 1). For those who may not be too familiar with it let me clarify the mathematical conjuring tricks which resulted in that figure. Impact factors are derived by dividing all the citations of the previous 2 years by the number of articles published in a given journal. There are a

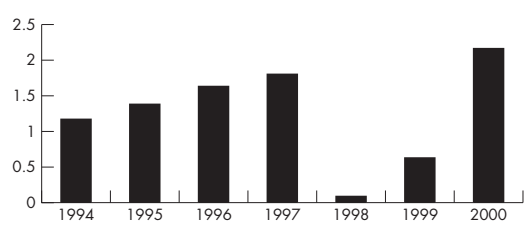

Figure 1 Impact factor of STI. few exceptions. For example, letters count as citations but not as articles. When conference abstracts are cited, an increasing and questionable practice, they are considered bona fide citations though the original abstract is not counted as a publication. The same is true for supplements.

You can see where this illogical juggling leads: journals with a large correspondence, or which publish conference abstract and supplements do well. More questionably, clinical journals do worse than pure science journals. This is because clinical research takes longer to perform than laboratory based research. Hence the "impact" of clinical studies is longerand certainly way beyond the arbitrary 2 year cutoff point. The final point to make is that the impact factor usually reflects the "impact" of one or two articles with high citations and is therefore more realistically the impact factor of an article rather than the journal as a whole. As you see, this is an imperfect measure of the quality of a journal. But it is all we have. The Americans, rightly I think, ignore it. The rest of the world are unnaturally wedded to it.

Sex Transm Inf 2002;78:6 